\title{
Algunas reflexiones sobre la equidad en la educación y su contexto en México Some reflections on the equity and its context in México
}

\author{
Carlos Miguel Amador Ortíz \\ Instituto Tecnológico Superior de Puerto Vallarta, México \\ carlos.amador@tecvallarta.edu.mx
}

\section{Resumen}

En este trabajo se hace una reflexión sobre el concepto de equidad a partir de la teoría de la justicia social de Rawls (1993), partiendo de una visión general sobre el panorama en América Latina y las actuales reformas en México; asimismo, se analizan algunos programas gubernamentales cuyo objetivo es contribuir a mejorar la equidad en México con base en los criterios establecidos por el Banco Mundial, específicamente los factores extraescolares que inciden en el éxito escolar y que impactan directamente en la equidad educativa (Aguerrondo, 1993). Los programas que se analizan en este trabajo son: escuelas dignas, escuelas de excelencia para abatir el rezago educativo, escuelas de tiempo completo (ETC), inclusión y alfabetización digital, e inclusión y equidad educativa.

Palabras clave: equidad, educación, calidad, justicia social, programas de intervención.

\section{Abstract}

This paper presents a reflection on the concept of equity from the theory of social justice proposed by Rawls (1993), based on an overview of the situation in Latin America, and the ongoing reforms in Mexico; also are described some government programs developed with the aim of contributing to improving equity in Mexico and analyzed based on the criteria established by the World Bank on school factors that influence school success and that directly impact on equity in Education (Aguerrondo, 1993). The programs discussed in this paper are: schools with 
dignity, schools of excellence to bring down the educational gap, full-time schools, inclusion and digital literacy and inclusion and educational equity.

Key words: equity, education, quality, social justice, intervention programs.

Fecha Recepción: Agosto $2015 \quad$ Fecha Aceptación: Diciembre 2015

\section{Introducción}

El actual panorama internacional presenta diferencias heterogéneas entre los países en cuanto a organización y estructura social, economía y política; aunque el sistema predominante en la esfera global es una economía de libre mercado, y la organización política generalizada proviene de las ideas de la ilustración con valores como la democracia y las libertades, el grado de introyección de estos valores es muy variable en las distintas regiones del mundo.

La situación de Latinoamérica se caracteriza por democracias en proceso de consolidación; los países latinoamericanos suelen ser políticamente estables y poseer instituciones consolidadas (aunque sigue sin solucionarse el problema crónico de la corrupción); sin embargo, al mismo tiempo tienen el problema de pobreza que experimenta la mayor parte de su población. De esa manera, estos países son considerados pobres o en vías de desarrollo y su economía crece de manera reducida en comparación con las de otras regiones.

Lo que invierten en el área educativa es poco comparado con lo que invierten los países desarrollados. El acceso a mejores oportunidades, servicios y calidad de vida solo se ofrece a ciertos grupos de la población, de algunas regiones de cada país (por ejemplo, la situación de pobreza y marginación es más extendida en poblaciones indígenas y en zonas rurales en comparación con la población no indígena y con las zonas urbanas).

Aunado a esto, en las últimas décadas ha habido un profundo cambio social, económico y político, pero los sistemas educativos no han ido a la par con ese cambio. A este respecto, autores como Braslavsky y Cosse (1996), mencionan que el tema educativo está en crisis, ocasionada por la expansión masiva del sistema educativo, falta de orientación hacia el aprendizaje continuo, 
desigualdades sociales, cambios en la familia, y desestructuración de los ejes del sistema educativo (secuencialidad y jerarquización).

Asimismo, Krawczyc (2002) menciona, tras analizar un conjunto de documentos oficiales de organismos multilaterales: del programa de promoción de la reforma educativa en América Latina y el Caribe (PREAL), del Banco Mundial (BM), de la Comisión Económica para América Latina y el Caribe (CEPAL) división de desarrollo social de la ONU, del Banco Interamericano de Desarrollo y del Instituto Internacional de Planeación de la Educación de la UNESCO (IIEPE), que existe un consenso entre los autores respecto a que los sistemas educativos vigentes - de la mayoría de los países- no están respondiendo a las demandas generadas por la reorganización de los sistemas democráticos y la apertura de la economía.

Con referencia a esta situación mundial, Cohen (2002) menciona que la equidad enfrenta algunas dificultades: no se aborda el tema desde la comprensión de la multiplicidad de factores explicativos, hay pocas evaluaciones sistemáticas de los proyectos implementados, y existe poca conexión entre la información disponible y los equipos que definen las políticas.

\section{DESARROLLO}

\section{El tema de la equidad en el marco de las democracias en América Latina}

El tema de la equidad conlleva de fondo un aspecto ideológico sobre el concepto de igualdad, como un valor que es concebido de una determinada forma de acuerdo al tipo de organización social y del consenso social predominante; algunos de los pilares de las sociedades modernas son la igualdad ante la ley, así como el ejercer derechos y deberes en un entorno de libertades civiles, políticas y económicas (Rodríguez, 2008).

Sin embargo, este entorno de libertades en una estructura social heterogénea conlleva una diferenciación entre las capacidades, oportunidades y acceso a mejores bienes, servicios y opciones de vida, que traen como consecuencia la generación de desigualdades a nivel social, y el problema ético de la justicia social.

Rodríguez (2008), analiza la concepción de Amartya Sen sobre la justicia social, partiendo de que toda concepción de equidad supone la definición de una igualdad fundamental que pudiese "justificar" otros tipos de desigualdades; en las sociedades modernas la igualdad fundamental es 
la igualdad de oportunidades e igualdad ante la ley, que en la actualidad se mantiene como un ideal utópico que no ha tenido resultados benéficos de carácter económico y social para las mayorías (al menos en América Latina).

Una de las propuestas actuales sobre injusticia social, enfocada en la igualdad, es la de Rawls (1993), quien parte de un principio hipotético al que denomina velo de la ignorancia, mencionando que si todos como sociedad estuviéramos en una posición de no conocer el lugar que debemos ocupar dentro de la estructura social (una estructura con diferencias económicas, culturales y de oportunidades, donde la mayoría está en desventaja) y tuviéramos que negociar una forma de organización social partiendo de ese principio, la mejor opción sería buscar un sistema más justo e igualitario para todos. A este respecto, Rawls propone dos principios de justicia:

1. Cada persona debe tener un derecho igual al esquema más extenso de libertades básicas iguales compatible con un esquema similar de libertades para otros, y

2. Las desigualdades sociales y económicas deben de resolverse de tal modo que: -resulten del mayor beneficio para los miembros menos aventajados de la sociedad (el principio de la diferencia).

-los cargos y puestos deben estar abiertos para todos bajo condiciones de igualdad de oportunidades (justa igualdad de oportunidades).

Dicha propuesta teórica sobre equidad es importante porque su punto de partida es la igualdad de derechos y libertades, así como la búsqueda del beneficio de los miembros menos aventajados; es decir, establece que debe haber un sistema de compensación en el que los más aventajados contribuyan a mejorar las condiciones de vida y oportunidades de los menos aventajados (mejorar la equidad).

Por tanto, como ya se mencionó, el principio de libertades de las sociedades modernas conlleva la generación de desigualdades en la distribución de la riqueza, las cuales son más marcadas en los países menos industrializados y en vías de desarrollo como los latinoamericanos. Vakis, Rigolini, y Luchetti (2015), citan datos de 2012 del Banco Mundial, donde se informa que $12.3 \%$ de la población de América Latina vivía en pobreza extrema (con \$2.5 dólares al día), 25.3 \% vivía en 
pobreza moderada (con $\$ 4$ dólares al día), $37.8 \%$ son vulnerables a la pobreza (con \$4 a \$10 dólares al día), y $34.2 \%$ pertenecía a la clase media (\$10 a \$50 dólares al día).

Por tanto, $75.4 \%$ de la población de América Latina en el año 2012 era considerada pobre o vulnerable a la pobreza, situación relacionada con falta de oportunidades, en un contexto social poco favorable para el desarrollo óptimo de los individuos, que aunado a una educación pública de baja calidad, se ha vuelto un círculo vicioso que impide mejorar las condiciones de equidad y de vida de las clases más desfavorecidas.

Con respecto a la influencia del contexto social en el tema educativo, resulta interesante observar los datos proporcionados por el Banco de Mundial (Aguerrondo, 1993): $60 \%$ del rendimiento escolar proviene de factores extraescolares de carácter socioeconómico y familiar (40 a $50 \%$ ), siendo el más importante el clima educacional dentro del hogar (el tiempo que dedicaron al estudio los adultos del hogar); la capacidad económica (25 a $30 \%$ ); y por último, el nivel de organización familiar.

Ante este panorama, donde se han tomado en cuenta tanto los factores que influyen en el rendimiento escolar, como la desigualdad y pobreza en América Latina, es importante elaborar una propuesta fundamentada en un análisis crítico de la realidad que de verdad incursione en la situación de equidad en el tema educativo. Retomando los postulados de la teoría de Rawls sobre justicia social, dicha propuesta debería enfocarse en apoyar a los menos aventajados en los elementos que están provocando una desigualdad de oportunidades. Así, el estudio de los factores extraescolares cobra importancia.

En respuesta a estas problemáticas, Cohen (2002) menciona un principio de equidad (congruente con el segundo principio de la teoría de la justicia social de Rawls), donde menciona que para superar las diferencias debe tratarse desigualmente a quienes son socioeconómicamente desiguales (acción afirmativa o discriminación positiva); es decir, es necesario intervenir en el tema educativo brindando una educación diferenciada de mayor calidad a los sectores más desfavorecidos, así como proponer estrategias para contrarrestar los efectos de los aspectos contextuales. 


\section{Algunos indicadores del tema educativo en México}

En México se ha incrementado notablemente el acceso al nivel de educación básica, es decir, primaria y secundaria: cerca del $100 \%$ de la población asiste a dicho nivel (INEE, 2013; Navarro, 2006); sin embargo, existe un rezago muy notable en otros grupos poblacionales de diferentes niveles y edades, donde el porcentaje de cobertura es menor.

Tabla I. Porcentaje de asistencia a la escuela

\begin{tabular}{|l|l|}
\hline Edades & Porcentaje \\
\hline 3 a 5 años & $73 \%$ \\
\hline 6 a 11 años & $98 \%$ \\
\hline 12 a 14 años & $93.3 \%$ \\
\hline 15 a 17 años & $71.1 \%$ \\
\hline 18 a 24 años & $32.2 \%$ \\
\hline
\end{tabular}

Fuente: (INEE, 2013).

De acuerdo a los datos del INEE (2013), tres de cada diez niños no asisten a preescolar y tres de cada diez jóvenes no asisten al bachillerato, cifra que se eleva aún más en el nivel superior, con siete de cada diez jóvenes que no acceden a la educación superior. La población que no asiste a la escuela asciende a 4.3 millones de personas en edades de 3 a 17 años.

Los índices de asistencia a la escuela de acuerdo a las condiciones económicas, según el INEE (2013), son: en preescolar (3 a 5 años de edad), de 79.6 \% en la población considerada no pobre y de $66.4 \%$ en la población pobre. La desigualdad es aún más notoria si se compara la población económica en el quinto quintil de ingreso (la población con mayores ingresos), que registra una asistencia de $89.3 \%$ en comparación con $66.4 \%$ de la población en pobreza alimentaria y $67.8 \%$ de la población en el primer quintil de ingreso (la población con menores ingresos). En la población de 15 a 17 años la desigualdad en el acceso incrementa significativamente con $77.6 \%$ en población no pobre respecto a $57.2 \%$ de asistencia de la población en situación de pobreza alimentaria, y comparando por el quintil de ingreso, el acceso es de $82.6 \%$ de la población en el quinto quintil y de $54.7 \%$ en la población del primer quintil de ingreso (ver gráfico 1). 


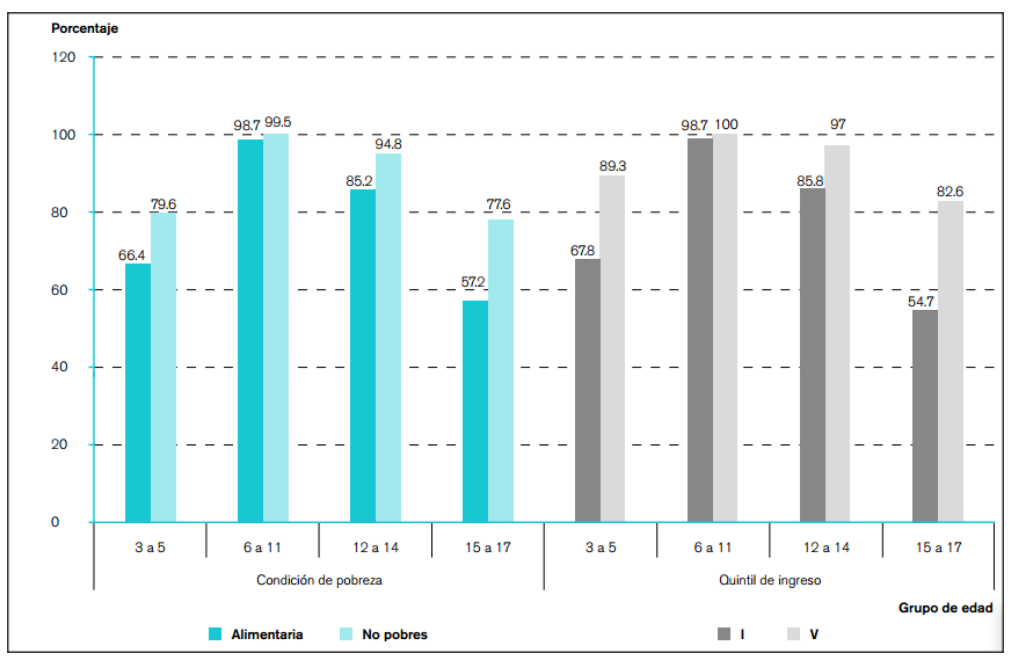

Gráfico 1. Porcentaje comparativo de asistencia a la escuela de grupos de población pobres y no pobres (INEE, 2013).

Las mayores desigualdades entre las poblaciones más vulnerables y las más beneficiadas con respecto a su asistencia al nivel de educación media superior se presentan en los hogares cuya cabeza de familia ha alcanzado un nivel de escolaridad de educación superior (96.1\%), o trabaja más de 20 horas semanales extra-domésticamente (30.5\%) (INEE, 2013).

Otro dato notable es las desigualdades por sexo, aspecto donde los hombres muestran desventaja con respecto a las mujeres en todos los niveles, inclusive en la educación superior (Bustos 2003).

Otro aspecto relevante en el tema del acceso educativo es el número de años de estudio en la población de 15 años de edad. Si se hace la comparación entre las localidades de zonas rurales, el promedio de años de estudio es de 6.4 y en zonas urbanas de 9.8; en la población indígena el promedio de años es de 6.4 y en la población no indígena de 9.1; por último, en la población con marginación alta el promedio es de 5.9 años y en la población con marginación baja de 9.1 (ver gráfico 2). 


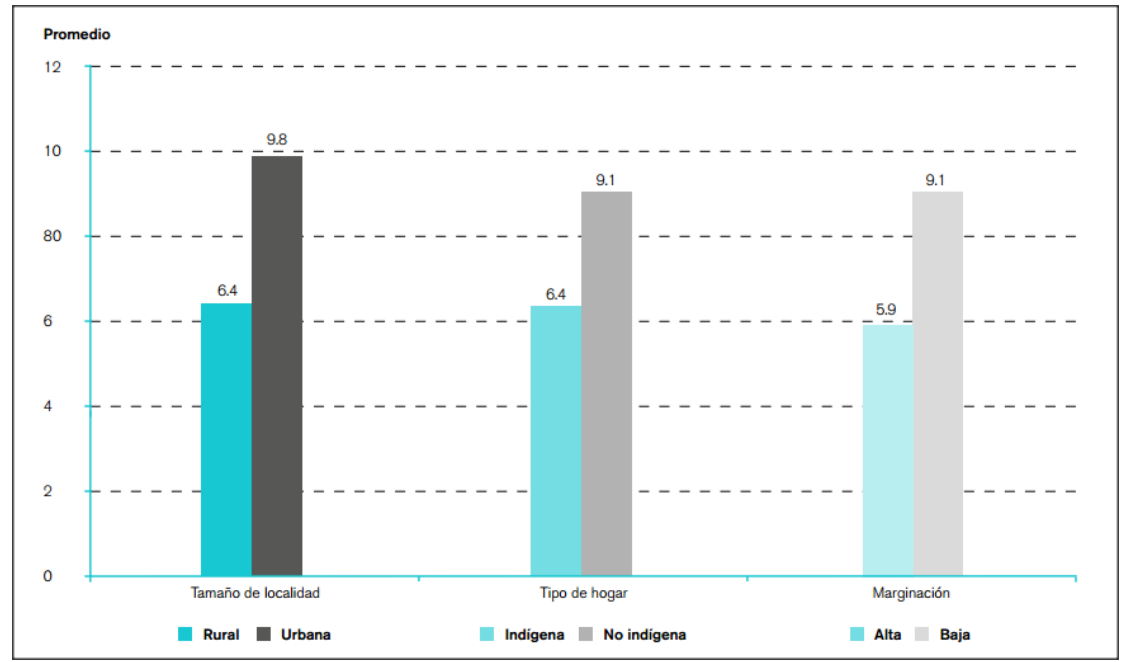

Gráfica 2. Escolaridad media de la población de 15 años de edad o más por tamaño de localidad, tipo de hogar y marginación (INEE, 2013).

\section{La equidad en las actuales reformas en México}

En México, al igual que en América Latina, el poder efectuar reformas educativas implica una labor ardua de negociación entre el Estado y los sindicatos, ya que estos últimos tienen un alcance nacional (Navarro, 2006); y por tanto, el proceso de formulación de políticas (PFP) se realiza principalmente mediante la negociación de estos dos actores (aun cuando puede existir participación de otros, tales como docentes, directores, supervisores e inclusive padres de familia, las negociaciones más importantes se dan entre sindicatos y el Estado).

En un entorno en el que se ha avanzado de forma notable en el acceso a los servicios educativos, el tema de la calidad y la equidad se torna más importante (Navarro, 2006); dicho tema en el marco de las reformas es de debate entre el sindicato y el Estado por las implicaciones que conlleva para los profesores ajustar los lineamientos de su función hacia los indicadores de calidad educativa. En México, a pesar de la resistencia de un amplio sector sindical y de docentes, se aprobó una reforma en 2012, la cual contempla dentro de sus aspectos más relevantes una modificación a los artículos de la Constitución política $3^{\circ}$ y $73^{\circ}$, donde se menciona que además de ser obligatoria, laica, gratuita y pública, la educación básica en México también debe ser de calidad e impartirse con equidad. 
Así, se establece que el Estado tiene la obligación no nada más de ofrecer educación, sino de que esta cumpla con los criterios cuantitativos de acceso y los cualitativos que garanticen a los estudiantes adquirir los conocimientos esperados del sistema y que les sirven para adaptarse y desenvolverse en el contexto actual. Cabe mencionar que el reto es verdaderamente grande y que aunque el objetivo puede parecer irreal en el corto plazo, sobre esta nueva base normativa se espera que se dirijan los esfuerzos del sistema para que se cumpla cabalmente con este nuevo derecho constitucional.

Uno de los elementos más destacables de esta reforma que se espera incidan favorablemente en la calidad y la equidad de la educación, es el mérito profesional como única forma de ingresar, permanecer y ascender como maestro, director o supervisor (tema que ha generado gran controversia y resistencia por parte de los sindicados docentes). Así, se han concedido facultades al Instituto Nacional para la Evaluación de la Educación (INEE) con el fin de que se tenga plena autonomía y se establezcan criterios y mecanismos que evalúen al personal docente y gestionen la profesionalización.

Uno de los objetivos fundamentales de la Reforma educativa es asegurar mayor equidad en el acceso a una educación de calidad. Con respecto a este objetivo se describen y analizan a continuación algunos programas de la Secretaría de Educación Pública orientados al tema de la equidad:

\section{Programa de escuelas dignas}

Este proyecto tiene como finalidad la rehabilitación de planteles escolares, y pretende consolidar la infraestructura escolar como un aspecto fundamental de la educación pública. Está enfocado hacia los planteles más desfavorecidos, específicamente a los ubicados en poblaciones indígenas y zonas rurales; se compone de fondos federales y estatales y brinda atención a los planteles en función de un diagnóstico técnico (Secretaría de Educación Pública, 2015).

El proyecto tiene como objetivo la rehabilitación de las siguientes áreas en las escuelas:

1. Seguridad estructural y condiciones generales de funcionamiento

2. Servicios sanitarios

3. Mobiliario y equipo

4. Accesibilidad

5. Áreas de servicios administrativos 
6. Infraestructura para la conectividad

7. Espacios de usos múltiples

\section{Escuelas de excelencia para abatir el rezago educativo}

El Programa Escuelas de Excelencia para Abatir el Rezago Educativo está orientado a emprender acciones que contribuyan a la disminución del rezago en las condiciones físicas de las escuelas públicas de educación básica y al fortalecimiento de la autonomía de gestión para mejorar la prestación del servicio educativo (Secretaría de Gobernación, 2015).

El programa está dirigido a las escuelas públicas de educación básica de los niveles de primaria y secundaria que se encuentran en condiciones de mayor rezago en cuanto a instalaciones y equipamiento según información del Censo de Escuelas, Maestros y Alumnos de Educación Básica y Especial (CEMABE), así como de las supervisiones de zona que atienden a estas escuelas

Con este programa la comunidad escolar recibe el recurso, planea y decide en colectividad sobre qué debe invertir primero: aulas, baños, bibliotecas, mobiliario del aula, espacios de usos múltiples, bardas, materiales educativos, capacitación a docentes y padres, computadoras, etcétera.

\section{Escuelas de Tiempo Completo (ETC)}

Este programa consiste en extender la jornada escolar en las instituciones públicas de educación básica para ampliar las oportunidades de aprendizaje de niños y adolescentes con el fin de mejorar los resultados educativos, fortalecer el desarrollo del currículo, propiciar el logro de aprendizajes con calidad en un marco de equidad, y atender las dificultades y necesidades educativas de todos los alumnos que asisten a ellas (Secretaría de Educación Pública, 2015).

Las ETC brindan el servicio educativo en los mismos 200 días lectivos que los demás planteles, pero durante una jornada extendida. Este programa va dirigido a los planteles más desfavorecidos: indígenas y rurales. 


\section{Inclusión y alfabetización digital}

Este programa consiste en proporcionar de forma gratuita tabletas electrónicas a alumnos de quinto grado de escuelas públicas de educación primaria para su uso personal y el de sus familias. Tiene como objetivo dotar de recursos tecnológicos a los niños para mejorar sus condiciones de estudio, reducir las brechas digitales y sociales de su familia y las de su comunidad, así como fortalecer y actualizar las formas de enseñanza de los maestros (Secretaría de Educación Pública, 2015).

Este programa va dirigido a los alumnos que cursan el $5^{\circ}$ grado de primaria en escuelas públicas ubicadas en el Distrito Federal.

\section{Inclusión y equidad educativa}

El programa para la inclusión y la equidad educativa tiene como objetivo contribuir a asegurar mayor cobertura, inclusión y equidad educativa entre todos los grupos de la población para la construcción de una sociedad más justa mediante normas y apoyos para los servicios educativos públicos, así como mejorar la infraestructura y equipamiento de instituciones públicas de educación básica, media superior y superior, que atienden población en contexto de vulnerabilidad y/o discapacidad (Secretaría de Gobernación, 2013).

Este programa impacta a los diferentes niveles educativos y aporta diferentes apoyos de acuerdo a cada nivel. En el nivel básico ofrece los siguientes apoyos:

\section{Apoyos técnicos}

1. Se proporcionan normas a las dependencias estatales de educación y, a solicitud expresa, asesoría y acompañamiento técnico, así como apoyo para el desarrollo de competencias locales que faciliten el cumplimiento de los objetivos y la evaluación del programa.

2. Se proporciona a la población objetivo asesoría y apoyo de las dependencias estatales en cada entidad federativa para fortalecer las capacidades de gestión (planeación, evaluación interna y seguimiento) de la comunidad escolar, propiciar condiciones de participación del alumnado, personal docente, madres y padres de familia, o tutores y la comunidad en general para mejorar los índices de permanencia, inclusión y logro de aprendizajes en educación inicial y básica, así 
como para integrar en su ruta de mejora escolar la atención de las prioridades educativas establecidas en el sistema básico de mejora.

\section{Apoyos financieros}

a) Se fortalecen los servicios de educación indígena, migrante y multigrado.

b) Se fortalecen los servicios de educación especial que atiende al alumnado con discapacidad o con aptitudes sobresalientes, mediante la transferencia de recursos a las entidades federativas para realizar actividades académicas y de vinculación interinstitucional; dotar de material didáctico y mobiliario o utensilios adaptados; realizar encuentros de redes de madres y padres de familia o tutores; implementar acciones de fortalecimiento académico y evaluación.

3. Se fortalecen las escuelas telesecundarias de las 32 entidades federativas con acciones de apoyo a la inclusión y la retención mediante transferencias de recursos para el seguimiento a la mejora continua de los procesos de estudio, evaluación local sobre la mejora en los niveles de logro de aprendizaje y desarrollo de estrategias al uso de los materiales educativos multimedia en los procesos de estudio del alumnado de telesecundarias.

4. Se brinda apoyo financiero para proyectos locales de fortalecimiento de inclusión y equidad educativa en el marco de los derechos humanos y la perspectiva de igualdad de género.

En educación media superior, los recursos financieros otorgados a través del programa se aplican en adquisición o pago de:

a) Computadoras y su equipo periférico, como impresoras, lectores ópticos, escáner, reguladores de voltaje y multicontactos, entre otros.

b) Programas de cómputo de uso general, incluyendo procesadores de texto, hojas de cálculo, bases de datos y demás que se requieran para la formación de los estudiantes con discapacidad, así como programas de protección contra virus y demás utilerías que sean necesarios.

c) Programas de cómputo especializados para apoyar las actividades de aprendizaje que realicen los usuarios por tipo de discapacidad, como visual, auditiva o motriz. 
d) Impresoras Braille, sistemas de audio y de proyección, así como cualquier otro dispositivo para apoyar las actividades de aprendizaje de los estudiantes con discapacidad de los Centros de Aprendizaje para Estudiantes con Discapacidad (CAED).

e) Mobiliario para la instalación y protección del equipo de cómputo.

f) Servicios inherentes al CAED, gastos de operación y sostenimiento de los CAED.

g) Las adecuaciones físicas necesarias que permitan la accesibilidad al CAED.

h) Mantenimiento del CAED.

Para el nivel educativo de educación superior, los tipos de apoyo que se proporcionan a través de este programa son:

1) Apoyos para el fortalecimiento de las Instituciones Públicas de Educación Superior (IPES) para la formulación de acciones que contribuyan a reducir las brechas de acceso a la educación a través de una amplia perspectiva de inclusión con enfoque de igualdad de género de personas en situación vulnerable.

2) Apoyos a las IPES que atienden a personas con discapacidad para dar respuesta a sus necesidades específicas, así como minimizar las barreras para el aprendizaje e impulsar la participación social. El apoyo consiste en la transferencia conforme a la disponibilidad presupuestaria del $100 \%$ de recursos presupuestados a la SES y asignados a las IPES para realizar actividades académicas, de vinculación, infraestructura, mobiliario y equipamiento para personas con discapacidad. 


\section{CONCLUSIÓN}

Una de las críticas mejor fundamentadas al sistema de organización política, económica y social predominante (democracias de libre mercado), es la polaridad social en el tema de la igualdad de oportunidades y de la distribución de los recursos. Esta polaridad tiende a acentuarse en los países más pobres y menos desarrollados.

La educación es uno de los elementos de los sistemas de organización social que puede contribuir a mejorar la igualdad de oportunidades que permitan a los sectores más vulnerables y marginados de la población acceder a mejores opciones de vida, y disminuir la desigualdad en la distribución de los recursos (es decir, la educación "puede y debe" ser el medio para brindar justicia social en un sistema de libertades que naturalmente genera desigualdades). Sin embargo, la interrogante que falta por contestar es: ¿Qué estructura y funciones debe poseer el sistema educativo para contribuir a mejorar la igualdad de oportunidades y generar una distribución de los recursos más equitativa entre los diferentes grupos de población en un país?

Con una reflexión a priori, y desde un marco de referencia orientado hacia la calidad educativa, sustentado en un análisis crítico del qué y para qué de la formación para configurar las formas y los contenidos educativos, se proponen fundamentalmente tres acciones:

- Caracterizar los diferentes grupos de población, principalmente los marginados que presentan mayor rezago en acceso educativo y éxito académico.

- Identificar mediante sólidos estudios científicos los factores escolares y extraescolares que inciden en el éxito académico, y su grado de influencia.

- Y por último, diseñar estrategias dirigidas a las necesidades de los diferentes grupos de población de acuerdo a sus características particulares y los factores que inciden en el éxito académico.

Por último, haciendo un análisis de los cinco programas de gobierno enfocados a mejorar la equidad en la educación se pueden emitir las siguientes conclusiones.

- Tres programas tienen por objetivo mejorar las condiciones de infraestructura y están dirigidos a los grupos más marginados de la población, favoreciendo así la equidad entre los grupos más vulnerables. Estos programas son: el programa de escuelas dignas, el 
programa de escuelas de calidad para abatir el rezago educativo, y el programa de inclusión y equidad educativa.

- El programa de inclusión y alfabetización digital está dirigido únicamente a niños que estudian en escuelas públicas del Distrito Federal, no se discrimina entre grupos de población marginados y no marginados, y por la zona en que se lleva a cabo el programa tomando como base el contexto y la situación educativa diferenciada entre esta zona urbana y las poblaciones rurales, contribuye poco a favorecer a los grupos que más requieren apoyos para mejorar la igualdad y la equidad educativa.

- El programa de escuelas de tiempo completo es el que más se ajusta a una propuesta específica de acuerdo a las necesidades de los grupos de población hacia los que va dirigido, y es probablemente el que puede llegar a tener un mayor impacto para mejorar la equidad educativa ya que ofrece una formación diferenciada (horario extendido) para los grupos más vulnerables y marginados de la población.

- Los programas enfocados a proporcionar infraestructura cumplen una función de equidad muy básica y necesaria; sin embargo, no se abordan los factores que inciden en el éxito académico como un todo, ya que no contemplan acciones particulares en los aspectos extraescolares que de acuerdo a los datos del Banco Mundial pueden llegar a tener una mayor influencia que los únicamente académicos; por tanto, para que los programas puedan obtener el impacto deseado en la equidad educativa se requiere que además de intervenir en los factores académicos puedan también incidir en los aspectos extraescolares. 


\section{Bibliografía}

Aguerrondo, I. (1993). La calidad de la educación: ejes para su definición y evaluación. Revista Interamericana de Desarrollo Educativo, pp. 561-578.

Braslavsky, C., y Cosse, G. (1996). Las actuales reformas educativas en América Latina. Cuatro actores, dos lógicas y ocho tensiones. Buenos Aires: Programa de promoción de la Reforma Educativa en América Latina y el Caribe.

Cohen, E. (2002). Educación, eficiencia y equidad: una difícil convivencia. Revista Iberoamericana de Educación, pp. 105-124.

Didou, S. (2011). Cobertura y promoción de la equidad en el Sistema de Educación Superior en México. Perfiles Educativos, pp. 59-65.

Latapí, P. (2006). Reseña de "equidad, calidad e innovación en el desarrollo educativo nacional" de la Secretaría de Educación Pública. Revista Mexicana de Investigación Educativa, 693701.

Navarro, J. (2006). Dos clases de políticas educativas. La política de las políticas públicas. Programa de promoción de la reforma educativa en América Latina y el Caribe.

Rawls, J. (1993). Liberalismo Político. Nueva York: Columbia University Press.

Rodríguez, C. (2008). Equidad de la educación en México. Propuesta de un sistema de indicadores. Revista perspectivas sociales, pp. 55-78.

Secretaría de Gobernación (28 de 12 de 2013). Diario Oficial de la Federación. Recuperado el 05 de 12 de 2015, de ACUERDO número 711 por el que se emiten las Reglas de Operación del Programa para la Inclusión y la Equidad Educativa: ACUERDO número 711 por el que se emiten las Reglas de Operación del Programa para la Inclusión y la Equidad Educativa

Vakis, R., Rigolini, J., y Luchetti, L. (2015). Los olvidados, pobreza crónica en América.

Bustos Romero, O. (2003). Mujeres y educación superior en México. Recomposición de la matrícula universitaria a favor de las mujeres. Repercusiones educativas, Económicas y $\begin{array}{llll}\text { Sociales. } & \text { México. } & \text { Obtenido }\end{array}$ http://www.mexicoconectado.gob.mx/images/stories/Liferay/eMex/informacion/ 
Gobierno de la República, México (2014). Reforma Educativa, resumen ejecutivo. México: Gobierno de la República. Obtenido de http://reformas.gob.mx/wpcontent/uploads/2014/04/RESUMEN_DE_LA_EXPLICACION_REFORMA_EDUCATIVA.pdf

INEE (2013). Panorama Educativo de México 2013. Indicadores del sistema educativo Nacional de Educación Básica y Media Superior. INEE. Obtenido de http://publicaciones.inee.edu.mx/buscadorPub/P1/B/112/P1B112.pdf

Krawczyc, N. (2002). La reforma educativa en América Latina desde la perspectiva de los organismos multilaterales. Revista Mexicana de Investigación Educativa, 627-673. Obtenido de http://www.redalyc.org/articulo.oa?id=14001609

Secretaría de Educación Pública (16 de 12 de 2015). Escuelas de tiempo completo. Recuperado el 17 de 12 de 2015, de Programa escuelas de tiempo completo: http://basica.sep.gob.mx/tiempocompleto/

Secretaría de Educación Pública (2015). Escuelas Dignas. Recuperado el 05 de 12 de 2015, de http://www.inifed.gob.mx/escuelas_dignas/

Secretaría de Educación Pública (13 de 08 de 2015). Programa de inclusión y alfabetización digital 2015 (PIAD). Recuperado el 05 de 12 de 2015, de http://www2.sepdf.gob.mx/principal/piad-2015-2016.jsp

Secretaría de Gobernación (28 de 02 de 2015). Diario Oficial de la Federación. Recuperado el 05 de 12 de 2015, de CONVENIO de Coordinación para la operación del Programa Escuelas de Excelencia para Abatir el Rezago Educativo: http://dof.gob.mx/nota_detalle.php?codigo $=5405514 \&$ fecha $=28 / 08 / 2015$ 